\title{
Endoscopic ultrasound-guided fine needle aspiration and endobronchial ultrasound-guided transbronchial needle aspiration: Are two better than one in mediastinal staging of non-small cell lung cancer?
}

\author{
Masahide Oki, MD, ${ }^{\mathrm{a}}$ Hideo Saka, MD, ${ }^{\mathrm{a}}$ Masahiko Ando, MD, ${ }^{\mathrm{b}}$ Chiyoe Kitagawa, MD, ${ }^{\mathrm{a}}$ \\ Yoshihito Kogure, MD, ${ }^{a}$ and Yukio Seki, MD
}

\begin{abstract}
Objective: The role of combined endobronchial ultrasound-guided transbronchial needle aspiration (EBUS-TBNA) and endoscopic ultrasound-guided fine needle aspiration (EUS-FNA) with a single bronchoscope is poorly understood. The purpose of the present study was to elucidate the roles of EBUS-TBNA and EUS-FNA with a single bronchoscope in the preoperative hilar and mediastinal staging of non-small cell lung cancer (NSCLC).
\end{abstract}

\begin{abstract}
Methods: A total of 150 patients with potentially resectable known or suspected NSCLC were enrolled in our prospective study. EBUS-TBNA was performed, followed by EUS-FNA, with an EBUS bronchoscope for N2 and $\mathrm{N} 3$ nodes $\geq 5 \mathrm{~mm}$ in the shortest diameter on ultrasound images, in a single session.

Results: EBUS-TBNA was performed for 257 lymph nodes and EUS-FNA for 176 lymph nodes. Of the 150 patients, 146 had a final diagnosis of NSCLC. Of these 146 patients, $33(23 \%)$ had N2 and/or N3 nodal metastases. The sensitivity of EBUS-TBNA, EUS-FNA, and the combined approach per patient was $52 \%$, $45 \%$, and $73 \%$, respectively (EBUS-TBNA vs the combined approach, $P=.016$, McNemar's test). The corresponding negative predictive value was $88 \%, 86 \%$, and $93 \%$. Two patients $(1 \%)$ developed severe cough from EBUS-TBNA.
\end{abstract}

Conclusions: The combined endoscopic approach with EBUS-TBNA and EUS-FNA is a safe and accurate method for preoperative hilar and mediastinal staging of NSCLC, with better results than with each technique by itself. (J Thorac Cardiovasc Surg 2014;148:1169-77)

Supplemental material is available online.

Endoscopic ultrasound (EUS)-guided needle techniques, including endobronchial ultrasound-guided (EBUS) transbronchial needle aspiration (EBUS-TBNA) and EUSguided fine needle aspiration (EUS-FNA), have been recommended as the test of choice for mediastinal staging of non-small cell lung cancer (NSCLC). ${ }^{1}$ Although either EBUS-TBNA $^{2,3}$ or EUS-FNA ${ }^{4,5}$ alone has been found to be an effective method, the combination of EBUS-TBNA and EUS-FNA has been reported to be more accurate than

\footnotetext{
From the Departments of Respiratory Medicine ${ }^{\mathrm{a}}$ and Thoracic Surgery, ${ }^{\mathrm{c}}$ Nagoya Medical Center, Nagoya, Japan; and Advanced Medicine and Clinical Research, ${ }^{\mathrm{b}}$ Nagoya University Hospital, Nagoya, Japan.

Disclosures: Authors have nothing to disclose with regard to commercial support. Received for publication Jan 7, 2014; revisions received April 29, 2014; accepted for publication May 6, 2014; available ahead of print June 13, 2014

Address for reprints: Masahide Oki, MD, Department of Respiratory Medicine, Nagoya Medical Center, 4-1-1 Sannomaru, Naka-ku, Nagoya 460-0001, Japan (E-mail: masahideo@aol.com).

$0022-5223 / \$ 36.00$

Copyright (c) 2014 by The American Association for Thoracic Surgery http://dx.doi.org/10.1016/j.jtcvs.2014.05.023
}

either method alone, ${ }^{6-10}$ because EBUS-TBNA and EUS-FNA have complementary roles for mediastinal exploration. ${ }^{11}$ However, the combination method has had some issues regarding the availability of expensive equipment and expertise. To overcome these problems, the utility of EUS-FNA with an EBUS bronchoscope in place of an EUS endoscope has been advocated. ${ }^{12-14}$ Although the procedure requires some experience and skill, it can be performed by a bronchoscopist with an EBUS bronchoscope and thus enable a simple combined transbronchial and transesophageal endoscopic approach. To date, a few investigators ${ }^{12,13}$ have suggested the efficacy of combined EBUS-TBNA and EUS-FNA with an EBUS bronchoscope in the mediastinal staging of NSCLC. However, because no prospective study has clearly demonstrated that the diagnostic value of the combined method is superior to that of each method alone, the roles remain unknown. The purpose of the present study was to elucidate the role of combined EBUS-TBNA and EUS-FNA with a single bronchoscope in preoperative hilar and mediastinal staging of NSCLC. The primary endpoint of the present study was to compare the diagnostic value of the combined method to that of each method by itself. The secondary endpoints were safety and the procedure duration. 


\section{Abbreviations and Acronyms \\ CT = computed tomography \\ EBUS = endobronchial ultrasound \\ EBUS-TBNA $=$ endobronchial ultrasound-guided transbronchial needle aspiration \\ EUS = endoscopic ultrasound \\ EUS-FNA $=$ endoscopic ultrasound-guided fine needle aspiration \\ NSCLC = non-small cell lung cancer \\ TBNA $=$ transbronchial needle aspiration}

\section{METHODS \\ Patients}

We performed a prospective study that had been approved by the institutional review board of Nagoya Medical Center (identifier, 2009-251) and registered with the University Hospital Medical Information Network-Clinical Trials Registry (identifier, UMIN000002882). From December 2009 to August 2012, 150 patients with potentially operable, pathologically proven or clinical or radiologically suspected, NSCLC were enrolled in the present study. The operability was decided from the radiologic findings, including chest computed tomography $(\mathrm{CT})$, positron emission tomography-CT, and brain magnetic resonance imaging, and the patients' condition. Patients with stage T4 or M1 disease according to the International Association for the Study of Lung Cancer staging system $^{15,16}$ were excluded. Patients with bulky N2 or N3 disease were also excluded. In our institution, we usually perform bronchoscopy for diagnosis and mediastinal staging in a separate setting; however, we sometimes perform EBUS-TBNA for highly suspicious mediastinal lymph nodes as an initial diagnostic test. Such patients with pathologically proven $\mathrm{N} 2$ or N3 disease were not included in the present study. All patients provided written informed consent. The baseline characteristics of the 150 patients are listed in Table 1.

\section{Procedures}

For EBUS-TBNA and EUS-FNA, a convex probe ultrasound bronchoscope (BF-UC260F-OL8 or BF-UC260FW; Olympus, Tokyo, Japan) and 22-gauge needles (NA-201SX-4022; Olympus) were used. The endoscopic procedures were performed with the patient under local anesthesia with lidocaine and conscious sedation with intravenous midazolam by staff pulmonologists or supervised pulmonary residents. EBUS-TBNA was performed first, followed by EUS-FNA, in a single session.

EBUS-TBNA was performed in the manner similar to the one we have previously described ${ }^{17}$ The procedure was performed with the patient in the supine position. After anesthetizing the upper airway with lidocaine, an EBUS bronchoscope was inserted into the trachea through the mouth, and lidocaine was administered into the trachea and bronchus through the working channel. Next, a balloon attached to the transducer was inflated with saline solution. It was then brought into contact with the airway wall and moved in all directions to identify the lesions for sampling. Once the target lesion had been visualized by ultrasound, a dedicated needle was passed through the working channel of the EBUS bronchoscope and advanced through the tracheobronchial wall into the lesion under real-time ultrasound visualization. After the central stylet had been removed, suction was applied using a syringe while manipulating the needle back and forth within the lesion. After sampling, the suction was released slowly, and the needle was retracted. The specimen collected in the lumen of the needle was first pushed out with the central stylet and then blown by air with a syringe onto a glass slide. The visible tissue
TABLE 1. Patient and lesion characteristics

\begin{tabular}{|c|c|}
\hline Characteristic & Value \\
\hline Patients (n) & 150 \\
\hline \multicolumn{2}{|l|}{ Gender } \\
\hline Male & $103(69)$ \\
\hline Female & $47(31)$ \\
\hline \multicolumn{2}{|l|}{$\operatorname{Age}(y)$} \\
\hline Mean \pm standard deviation & $68.3 \pm 8.6$ \\
\hline Range & $33-83$ \\
\hline \multicolumn{2}{|l|}{ Smoking history } \\
\hline Never & $30(20)$ \\
\hline Former & $52(35)$ \\
\hline Current & $68(45)$ \\
\hline \multicolumn{2}{|l|}{ Primary lesion location by bronchopulmonary segment } \\
\hline Right upper lobe & $51(34)$ \\
\hline Right middle lobe & $3(2)$ \\
\hline Right lower lobe & $24(16)$ \\
\hline Left upper lobe & $34(23)$ \\
\hline Lingula & $7(5)$ \\
\hline Left lower lobe & $31(21)$ \\
\hline \multicolumn{2}{|l|}{ Final histopathologic classification } \\
\hline \multicolumn{2}{|l|}{ Non-small cell lung cancer } \\
\hline Adenocarcinoma & $89(59)$ \\
\hline Squamous cell carcinoma & $48(32)$ \\
\hline Large cell carcinoma & $3(2)$ \\
\hline Adenocarcinoma + squamous cell carcinoma & $2(1)$ \\
\hline Adenocarcinoma + large cell carcinoma & $1(1)$ \\
\hline Squamous cell carcinoma + small cell carcinoma & $1(1)$ \\
\hline Adenocarcinoma + small cell carcinoma & $1(1)$ \\
\hline Sarcomatoid carcinoma & $1(1)$ \\
\hline \multicolumn{2}{|l|}{ Other } \\
\hline Small cell carcinoma & $2(1)$ \\
\hline Tuberculosis & $1(1)$ \\
\hline Organizing pneumonia & $1(1)$ \\
\hline \multicolumn{2}{|l|}{ Preprocedural diagnosis for non-small cell lung cancer } \\
\hline Diagnosed & $137(91)$ \\
\hline Undiagnosed, but suspected & $13(9)$ \\
\hline
\end{tabular}

Data presented as n (\%), unless otherwise noted.

fragment on the glass slide was then collected and transferred into numbered separate containers filled with formalin for histologic examination. The remaining specimen on the glass slide was smeared with another glass slide and fixed in $95 \%$ alcohol for cytologic examination. To clarify the role and diagnostic ability of each needle aspiration procedure, rapid on-site cytologic examination was not used. EBUS-TBNA was performed for N3 nodes, followed by the N2 nodes that were $\geq 5 \mathrm{~mm}$ in the shortest diameter on the ultrasound images. $\mathrm{N} 1$ nodes were examined after the N2 nodes if the attending physician or examiner considered it necessary. Two punctures were made for each lymph node, as previously reported by Herth and colleagues. ${ }^{18}$ The lymph node location examined and the duration of the procedure from insertion to removal of an EBUS bronchoscope were recorded.

After EBUS-TBNA, EUS-FNA was performed at the left lateral position, as previously described. ${ }^{19}$ An EBUS bronchoscope was inserted and advanced through the esophagus while examining the structure around the esophagus by ultrasound. Once the target lesion had been identified, it was punctured through the esophagus with another needle to avoid contamination from the EBUS-TBNA samples under real-time ultrasound guidance. Next, the needle was manipulated back and forth within the lesion 


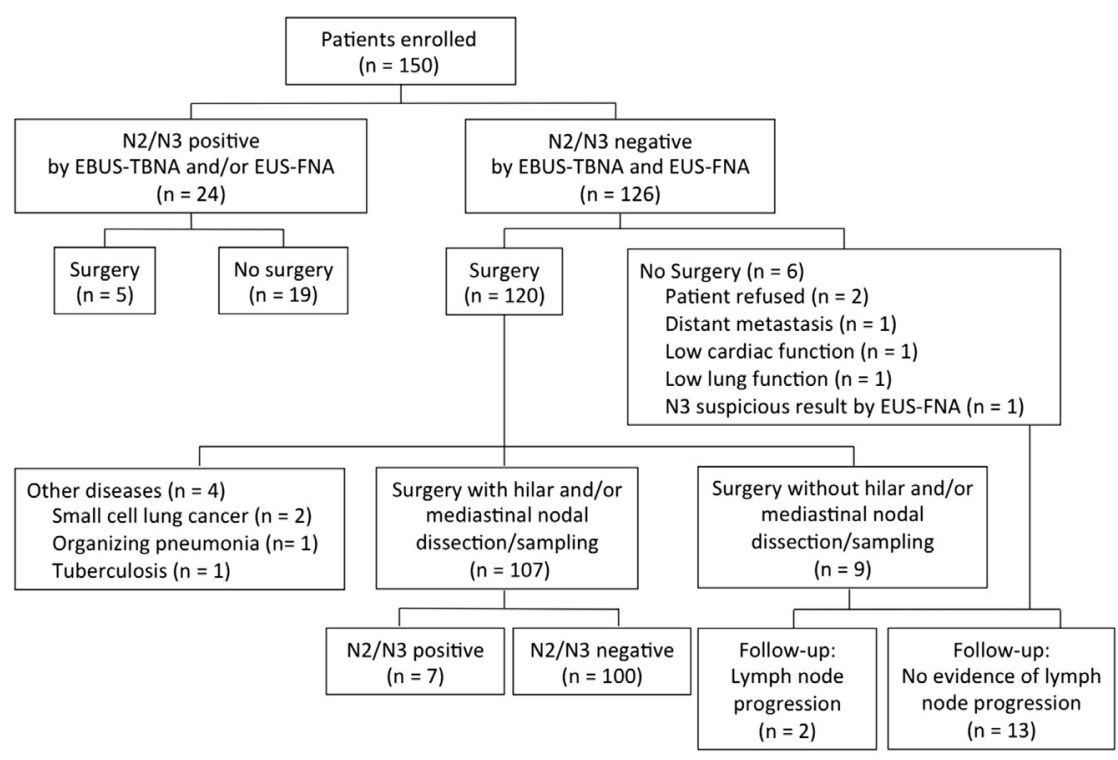

FIGURE 1. Clinical course of patients enrolled in the study. EBUS-TBNA, Endobronchial ultrasound-guided transbronchial needle aspiration; EUS-FNA, endoscopic ultrasound with bronchoscope-guided fine needle aspiration.

while applying suction under ultrasound guidance and then retracted to collect the aspirated specimen. The handling of the sampled specimens, the size criteria and order in each lymph node for needle aspiration, and the puncture number was the same as for the EBUS-TBNA procedure. To clarify the role and diagnostic ability of each procedure, EUS-FNA was performed even for lymph nodes that had been evaluated using EBUS-TBNA.

Surgical resection with lymph node dissection and/or examination was performed for patients with no evidence of $\mathrm{N} 2$ or $\mathrm{N} 3$ metastasis and for patients whose attending physician considered it appropriate. An experienced thoracic surgeon decided the operative procedure.

\section{Final Diagnosis}

The final diagnosis of lymph node metastases was established by the results of the surgical procedure, EBUS-TBNA and/or EUS-FNA, or radiologic evidence of lymph node progression. If no lymph node

TABLE 2. Locations of lymph nodes evaluated by EBUS-TBNA and EUS-FNA

\begin{tabular}{lcc}
\hline Lymph node location & EBUS-TBNA (n) & EUS-FNA (n) \\
\hline $2 \mathrm{R}$ & 12 & 0 \\
$2 \mathrm{~L}$ & 0 & 4 \\
$3 \mathrm{p}$ & 1 & 3 \\
$4 \mathrm{R}$ & 65 & 1 \\
$4 \mathrm{~L}$ & 56 & 66 \\
5 & 0 & 2 \\
7 & 77 & 79 \\
8 & 0 & 5 \\
$10 \mathrm{~L}$ & 10 & $16 \dagger$ \\
$11 \mathrm{R}$ & $20^{*}$ & 0 \\
11L & 16 & 0 \\
Total & 257 & 176 \\
\hline
\end{tabular}

EBUS-TBNA, Endobronchial ultrasound-guided transbronchial needle aspiration; EUS-FNA, endoscopic ultrasound-guided fine needle aspiration. *Included $2 \mathrm{~N} 1$ lymph nodes. †Included 3 N1 lymph nodes. progression was seen on $\mathrm{CT} \geq 6$ months after EBUS-TBNA and/or EUS-FNA, the lymph nodes were regarded as benign. ${ }^{20}$ Suspicious findings from the needle aspiration procedure were regarded as negative in our analysis. The positive findings from the needle aspiration procedure were regarded as true-positive results in our analysis, because the occurrence of false-positive results has been reported to be extremely rare.

\section{Statistical Analysis}

The sensitivity of EBUS-TBNA, EUS-FNA, and combined EBUS-TBNA and EUS-FNA approach had been assumed to be $70 \%$, $70 \%$, and $93 \%$, respectively, according to the findings from a previous study. ${ }^{8}$ From this information, we planned to accrue $\geq 129$ patients with malignancy to help us detect any superiority in the diagnostic sensitivity of the combined EBUS-TBNA and EUS-FNA procedure compared with a single procedure (EBUS-TBNA or EUS-FNA) at a significance level of 0.05 , with $80 \%$ statistical power. The homogeneity in the performance of the 2 diagnostic procedures was assessed using the exact McNemar test. Diagnostic sensitivity and the negative predictive value were calculated using the standard definitions, and the $95 \%$ confidence intervals were calculated based on the binomial distribution. The mean values and percentages are presented, as appropriate. Continuous variables were analyzed using the Mann-Whitney $U$ test. The $P$ value was 2-tailed. Statistical analyses were performed using a statistical software program (PASW Statistics, version 18; SPSS, Inc, Chicago, Ill).

\section{RESULTS \\ Patients}

Figure 1 and Figure E1 show the clinical course of the 150 patients enrolled in the present study. A total of $146 \mathrm{pa}-$ tients had a final diagnosis of NSCLC. Of these 146 patients, 121, including 5 with N2- and/or N3-positive results by EBUS-TBNA and/or EUS-FNA, underwent surgery. The surgical procedures were pneumonectomy with nodal dissection or sampling in 3, lobectomy with or without nodal dissection or sampling in 102, segmentectomy with or without nodal dissection or sampling in 6 , 
TABLE 3. Diagnostic values of EBUS-TBNA and EUS-FNA stratified by patient

\begin{tabular}{|c|c|c|c|c|c|c|}
\hline \multirow[b]{2}{*}{ Diagnostic value } & \multicolumn{2}{|c|}{ EBUS-TBNA } & \multicolumn{2}{|c|}{ EUS-FNA } & \multicolumn{2}{|c|}{ EBUS-TBNA + EUS-FNA } \\
\hline & n/Total $(\%)$ & $95 \% \mathrm{CI}$ & $\mathbf{n} /$ Total $(\%)$ & $\mathbf{9 5} \% \mathrm{CI}$ & $\mathrm{n} /$ Total $(\%)$ & $95 \% \mathrm{CI}$ \\
\hline Sensitivity* & $17 / 33(52)$ & $34-69$ & $15 / 33(45)$ & $28-64$ & $24 / 33(73)$ & $54-87$ \\
\hline Specificity & $113 / 113(100)$ & $97-100$ & $113 / 113(100)$ & $97-100$ & $113 / 113(100)$ & $97-100$ \\
\hline Positive predictive value & $17 / 17(100)$ & $81-100$ & $15 / 15(100)$ & $78-100$ & $24 / 24(100)$ & $85-100$ \\
\hline Negative predictive value & $113 / 129(88)$ & $81-93$ & $113 / 131(86)$ & $79-92$ & $113 / 122(93)$ & $86-97$ \\
\hline Accuracy & $130 / 146(89)$ & $83-94$ & $128 / 146(88)$ & $81-93$ & $137 / 146(94)$ & $89-97$ \\
\hline
\end{tabular}

EBUS-TBNA, Endobronchial ultrasound-guided transbronchial needle aspiration; EUS-FNA, endoscopic ultrasound-guided fine needle aspiration; CI, confidence interval.

*EBUS-TBNA versus combined approach, $P=.016$; EUS-FNA versus combined approach, $P=.004$; McNemar's test.

wedge resection with nodal dissection or sampling in 8 , and thoracotomy with mediastinal exploration in 2.

\section{EBUS-TBNA and EUS-FNA}

Two patients $(1 \%)$ in whom severe cough had developed during the EBUS-TBNA procedure could not undergo additional EBUS or EUS evaluation. No other complications, including hemorrhage, mediastinitis, or pneumothorax, were observed. The median dose of midazolam used was $4 \mathrm{mg}$ (range, 2-8). EBUS-TBNA was performed for 257 lymph nodes (median, $8.2 \mathrm{~mm}$ in the shortest diameter on CT; range, 3.4-17.1) in 121 patients. EUS-FNA was performed for 176 lymph nodes (median, $7.8 \mathrm{~mm}$; range, 4.1-17) in 107 patients. The lymph node locations sampled by the procedures are listed in Table 2.

In the 146 patients with NSCLC, $33(23 \%)$ were diagnosed with $\mathrm{N} 2$ or $\mathrm{N} 3$ disease. The final tumor and nodal stage and TNM classification determined from the final staging procedures (surgery, endoscopic needle aspiration, or radiologic findings) were as follows: $\mathrm{T} 1$ in $71, \mathrm{~T} 2$ in 55, T3 in 19, and T4 in 1; N0 in 103, N1 in 10, N2 in 30, and $\mathrm{N} 3$ in 3 ; stage IA in 57 , IB in 26 , IIA in 16 , IIB in 10 , IIIA in 29 , IIIB in 4 , and IV in 4.

The diagnostic values of the procedures per patient are summarized in Table 3. The diagnostic sensitivity of the combined approach was significantly greater than that of each procedure alone (EBUS-TBNA vs combined approach; $P=.016$, EUS-FNA vs combined approach; $P=.004)$. The details of the patients with mediastinal metastases diagnosed only by EUS-FNA and EBUSTBNA are listed in Tables 4 and 5, respectively. Representative patients diagnosed with mediastinal metastasis only by EUS-FNA are shown in Figures 2 and 3. Surgery alone revealed mediastinal metastases in an additional 7 patients (only micrometastases in 2). The lymph node locations were as follows: stations $2 \mathrm{R}$ in 1 , $4 \mathrm{R}$ and 7 in 1, 7 in 1, 5 in 3, and 6 in 1 . The positive yield of EBUS-TBNA and EUS-FNA according to lymph node size is presented in Table 6. The sensitivity of EBUS-TBNA and EUS-FNA stratified by lesion is provided in Table 7.

Of the 24 patients with $\mathrm{N} 2$ or $\mathrm{N} 3$ disease confirmed by EBUS-TBNA and/or EUS-FNA, 19 did not undergo surgical resection but received chemotherapy $(n=7)$ or chemoradiotherapy $(\mathrm{n}=12)$. The remaining 5 patients underwent surgical resection, followed by chemotherapy $(\mathrm{n}=3)$ or chemoradiotherapy $(\mathrm{n}=2)$.

The duration of the procedures is listed in Table 8 . When we examined $\leq 2$ lymph nodes, the duration of EUS-FNA was significantly shorter than that of EBUS-TBNA.

\section{DISCUSSION}

In the present study, we investigated the efficacy of combined EBUS-TBNA and EUS-FNA with a single bronchoscope in preoperative hilar and mediastinal staging of NSCLC. We demonstrated greater sensitivity with the combined approach than with either alone. In addition, the feasibility and safety were high. We were able to

TABLE 4. Details of 7 patients with mediastinal metastases diagnosed by EUS-FNA but not EBUS-TBNA

\begin{tabular}{|c|c|c|c|c|c|}
\hline Pt. no. & Age (y) & Gender & Histopathologic type & $\begin{array}{c}\text { Lymph node } \\
\text { location with positive EUS-FNA } \\
\text { results (shortest diameter on CT, mm) }\end{array}$ & EBUS-TBNA \\
\hline 1 & 76 & Male & Squamous cell carcinoma & $5(8.7), 7(7.5)$ & Not performed \\
\hline 2 & 64 & Male & Squamous cell carcinoma & $5(16.5)$ & Not performed \\
\hline 3 & 65 & Female & Squamous cell carcinoma & $7(17.0)$ & Negative \\
\hline 4 & 72 & Male & Squamous cell carcinoma & $7(9.6)$ & Negative \\
\hline 5 & 60 & Male & Squamous cell carcinoma & $2 \mathrm{~L}(9.5)$ & Not performed \\
\hline 6 & 79 & Male & Squamous cell carcinoma & 4L (7.4) & Negative \\
\hline 7 & 34 & Female & Adenocarcinoma & $7(7.8)$ & Negative \\
\hline
\end{tabular}


TABLE 5. Details of 9 patients with mediastinal metastases diagnosed by EBUS-TBNA but not EUS-FNA

\begin{tabular}{lcllcl}
\hline & & & & & $\begin{array}{c}\text { Lymph node location with } \\
\text { positive EBUS-TBNA results }\end{array}$ \\
Pt. no. & Age $(\mathbf{y})$ & Gender & Histopathologic type & (shortest diameter on CT, mm) & EUS-FNA \\
\hline 1 & 70 & Male & Adenocarcinoma & $4 \mathrm{R}(12.9)$ & Not performed \\
2 & 54 & Male & Non-small cell carcinoma & $4 \mathrm{R}(10.3), 7(9.5)$ & Not performed for 4R, suspicious result for 7 \\
3 & 66 & Male & Non-small cell carcinoma & $4 \mathrm{R}(10.3)$ & Not performed \\
4 & 69 & Female & Adenocarcinoma & $4 \mathrm{R}(14.2)$ & Not performed \\
5 & 61 & Male & Adenocarcinoma & $4 \mathrm{R}(9.9)$ & Not performed \\
6 & 70 & Male & Adenocarcinoma & $4 \mathrm{R}(6.0)$ & Not performed \\
7 & 69 & Female & Adenocarcinoma & $4 \mathrm{R}(6.0)$ & Not performed \\
8 & 63 & Male & Squamous cell carcinoma & $4 \mathrm{R}(10.9)$ & Not performed \\
9 & 76 & Male & Squamous cell carcinoma & $4 \mathrm{R}(13.8), 2 \mathrm{R}(9.7)$ &
\end{tabular}

complete the procedures in all but 2 patients, who had developed a bad cough during EBUS-TBNA.

The development of EUS endoscopes and EBUS bronchoscopes has dramatically changed the approach to mediastinal staging of NSCLC. Although controversial, ${ }^{21}$ several investigators have reported that the diagnostic sensitivity of EBUS-TBNA ${ }^{3,4}$ or EUS-FNA ${ }^{4,5}$ was similar or greater than that of mediastinoscopy, which has been considered the reference standard for mediastinal staging of lung cancer. Thus, EBUS-TBNA or EUS-FNA has become increasingly accepted as a staging procedure before surgical biopsy. ${ }^{1,22}$ A recent review study reported that the diagnostic sensitivity of EBUS-TBNA and EUSFNA was $89 \%$ (range, $46 \%-97 \%$ ) and $89 \%$ (range, 45\%-100\%), respectively. ${ }^{1}$ Either procedure alone seems sufficiently sensitive as a single method; however, the sensitivity is likely to be affected by the prevalence of the malignancy or suspected nodal locations accessible by each method. ${ }^{1}$ Thus, EBUS-TBNA, which can access the paratracheal, subcarinal, and hilar regions, and EUS-FNA, which can access the subcarinal, aortopulmonary window, and lower mediastinal regions, are complementary in the mediastinal staging of lung cancer. ${ }^{11}$ EBUS-TBNA and
EUS-FNA combined can access nearly all mediastinal lymph nodes. Several investigators ${ }^{6-10,23}$ have reported the usefulness of combined EBUS-TBNA and EUS-FNA. Wallace and colleagues ${ }^{8}$ compared the diagnostic accuracy of conventional TBNA, EBUS-TBNA, and EUS-FNA for mediastinal staging of lung cancer. These procedures were performed sequentially at the same session in 138 patients. ${ }^{8}$ The sensitivity of conventional TBNA, EBUSTBNA alone, EUS-FNA alone, and combined EBUSTBNA and EUS-FNA was $36 \%, 69 \%, 69 \%$, and $93 \%$, respectively. ${ }^{8}$ Szlubowski and colleagues ${ }^{9}$ investigated the diagnostic value of EBUS-TBNA and EUS-FNA in 120 patients with NSCLC with normal-size mediastinal nodes. The sensitivity of EBUS-TBNA, EUS-FNA, and combined EBUS-TBNA and EUS-FNA was $46 \%, 50 \%$, and $68 \%$, respectively (EBUS-TBNA alone vs combined EBUSTBNA and EUS-FNA, $P=.04$ ). Annema and colleagues ${ }^{23}$ conducted a randomized trial of 241 patients to compare surgical staging alone and combined EBUS-TBNA and EUS-FNA followed by surgical staging. The sensitivity of combined EBUS-TBNA and EUS-FNA followed by surgical staging was significantly greater than surgical staging alone $(94 \%$ vs $79 \%, P=.02)$. As these positive
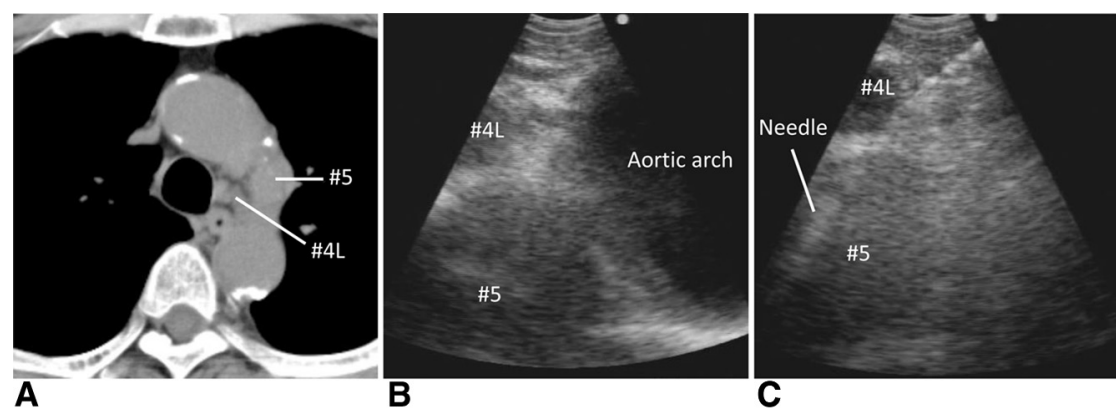

FIGURE 2. Transesophageal endoscopic ultrasound-guided fine needle aspiration for the subaortic lymph node (no. 5), which provided the only evidence of N2 disease (squamous cell carcinoma). The results of endobronchial ultrasound-guided transbronchial needle aspiration and transesophageal endoscopic ultrasound-guided fine needle aspiration for the left lower paratracheal lymph node (no. 4L) were both negative. A, Computed tomography image. B and C, Transesophageal endoscopic ultrasound images. 


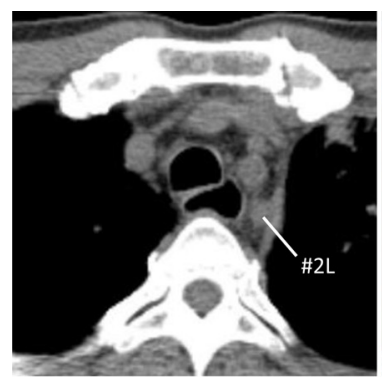

A

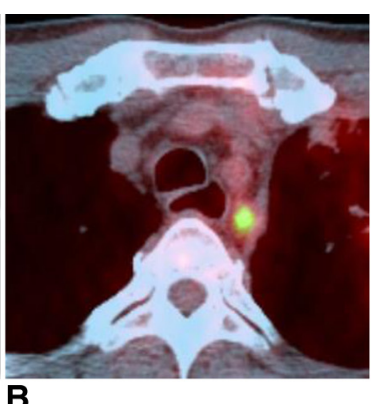

B

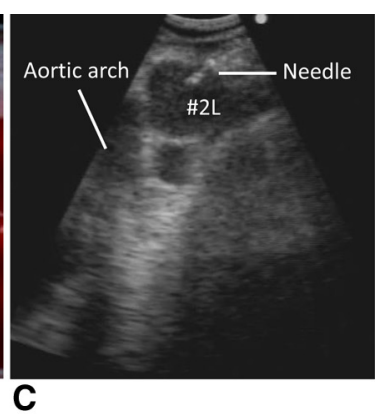

FIGURE 3. Transesophageal endoscopic ultrasound-guided fine needle aspiration for the left upper paratracheal lymph node (no. 2L), which provided the only diagnosis of N2 disease (squamous cell carcinoma). A, Computed tomography image. B, Positron emission tomography-computed tomography image. C, Transesophageal endoscopic ultrasound image.

results have emerged, the combined approach with EBUS-TBNA and EUS-FNA has been described as the best mediastinal staging procedure by endoscopy in recent review studies. ${ }^{22,24}$

Although the accuracy seems to be optimal, the combined EBUS-TBNA and EUS-FNA approach has a serious drawback: the necessity for both an EBUS bronchoscope and an EUS endoscope. Furthermore, most bronchoscopists might not be familiar with the handling of an EUS scope, adding the requirement for an additional experienced endoscopist to perform the combined procedure. The equipment and expertise would not be available in most institutions. In the technique described by Ohnishi and colleagues, ${ }^{10}$ each procedure can be performed separately at different specialized centers; however, that could lead to high costs and be time-consuming. To date, several investigators have demonstrated the feasibility, safety, and effectiveness of EUS-FNA with an EBUS bronchoscope for diagnosing benign ${ }^{19,25}$ and malignant ${ }^{12-14,26-28}$ disease. In the combined transbronchial and transesophageal approach, the use of the EBUS bronchoscope in place of the EUS endoscope for the transesophageal approach is more practical, because all procedures can be performed by a bronchoscopist using an EBUS bronchoscope. The combined approach using a single bronchoscope seems much simpler, more cost effective, ${ }^{29}$ and less-time consuming than the approach using both an EBUS bronchoscope and an EUS endoscope. To date, a few studies have reported on its usefulness for preoperative mediastinal staging of lung cancer. Hwangbo and colleagues ${ }^{13}$ reported the effectiveness of adding EUS-FNA with an EBUS bronchoscope to EBUS-TBNA in the mediastinal staging of NSCLC. In 150 patients with potentially operable lung cancer, EBUS-TBNA was performed, followed by EUS-FNA for the patients in whom the mediastinal lymph nodes were inaccessible or difficult to access using EBUS. The sensitivity, negative predictive value, and accuracy increased from $84 \%$ to $91 \%, 93 \%$ to $96 \%$ and $95 \%$ to $97 \%$ by adding EUS-FNA to EBUS-TBNA, respectively. No complication associated with EUS-FNA was observed in their study. ${ }^{13}$ Herth and colleagues ${ }^{12}$ investigated the feasibility and efficacy of EBUS-TBNA and EUS-FNA with a single bronchoscope for 150 patients with proven or suspected lung cancer with enlarged mediastinal lymph nodes. They also demonstrated that the combination of EBUS-TBNA and EUSFNA increased the diagnostic sensitivity compared with each method alone (EBUS-TBNA, 92\%; EUS-FNA, 89\%; combined approach, 96\%) without any complications. Although the sensitivity of the combined approach was greater than that of EBUS-TBNA alone in the studies by Hwangbo and colleagues ${ }^{13}$ and Herth and colleagues, ${ }^{12}$ the effect of adding EUS-FNA did not seem as large because of the high sensitivity of EBUS-TBNA alone. Our study has shown more clearly the greater effectiveness of adding EUS-FNA to EBUS-TBNA compared with previous studies.

The sensitivity of EBUS-TBNA and EUS-FNA in our study seemed to be lower than that in previous studies. The reasons might have been the low prevalence of

TABLE 6. Yield of EBUS-TBNA and EUS-FNA stratified by mediastinal nodal size on CT

\begin{tabular}{lccrrr}
\hline $\begin{array}{c}\text { Mediastinal lymph } \\
\text { nodes in shortest }\end{array}$ & & \multicolumn{3}{c}{ Patients with positive results (n) } \\
\cline { 3 - 6 } diameter on CT $(\mathbf{m m})$ & Patients (n) & Total with N2-N3 disease (n) & EBUS-TBNA & EUS-FNA & EBUS-TBNA + EUS-FNA \\
\hline$<10$ & 107 & 12 & $3(25)$ & $4(33)$ & $7(58)$ \\
$\geq 10$ & 39 & 21 & $14(67)$ & $11(52)$ & $17(81)$ \\
Total & 146 & 33 & $17(52)$ & $15(45)$ & $24(73)$ \\
\hline
\end{tabular}

Data in parentheses are percentages. EBUS-TBNA, Endobronchial ultrasound-guided transbronchial needle aspiration; EUS-FNA, endoscopic ultrasound-guided fine needle aspiration; $C T$, computed tomography. 
TABLE 7. Sensitivity of EBUS-TBNA and EUS-FNA per lesion

\begin{tabular}{lcccc}
\hline & $\begin{array}{c}\text { Total pathologically } \\
\text { Lymph node } \\
\text { proven malignant lesions by }\end{array}$ & \multicolumn{3}{c}{ Positive results (n)* } \\
\cline { 2 - 5 } & surgery or needle aspiration $(\mathbf{n})$ & EBUS-TBNA & EUS-FNA & EBUS-TBNA + EUS-FNA \\
\hline $2 \mathrm{R}$ & 5 & $4(80)$ & $0(0)$ & $4(80)$ \\
$2 \mathrm{~L}$ & 1 & $0(0)$ & $1(100)$ & $1(100)$ \\
$4 \mathrm{R}$ & 13 & $12(92)$ & $0(0)$ & $12(92)$ \\
$4 \mathrm{~L}$ & 4 & $1(25)$ & $4(100)$ & $4(100)$ \\
5 & 5 & $0(0)$ & $2(40)$ & $2(40)$ \\
6 & 1 & $0(0)$ & $0(0)$ & $0(0)$ \\
7 & 14 & $7(50)$ & $10(71)$ & $12(86)$ \\
Total & 43 & $24(56)$ & $17(40)$ & $35(81)$ \\
\hline
\end{tabular}

Data in parentheses are percentages. EBUS-TBNA, Endobronchial ultrasound-guided transbronchial needle aspiration; EUS-FNA, endoscopic ultrasound-guided fine needle aspiration. *Results of lesions without punctures during EBUS or EUS were regarded as negative.

malignancy $(23 \%)$, which has been reported to affect the sensitivity. ${ }^{1}$ In addition, our study included consecutive patients with or without enlarged mediastinal lymph nodes, regardless of the lymph node location. In fact, 4 of 7 patients, who had been diagnosed with $\mathrm{N} 2$ or $\mathrm{N} 3$ disease only by surgery, had single station 5 or 6 lymph node metastases. Other possible reasons include procedural or technical issues, such as the number of aspirations (2 aspirations per lesion in our study) or the level of the examiner's skill. Previous studies of EBUS-TBNA for mediastinal staging of NSCLC have recommended $\geq 2$ needle aspirations per lymph node station ${ }^{30}$ and $>2$ lymph node stations. ${ }^{31}$ However, optimal results were obtained by 3 needle aspirations ${ }^{30}$ and 4 lymph node stations ${ }^{31}$ in those studies.

EUS endoscopes have some distinct diagnostic advantages over EBUS bronchoscopes, including the availability of larger and longer needles, better visibility with the endoscope and ultrasound, with a wider ultrasound scanning range, and adjustability of the protruding needle angle using the elevator. These factors are why conventional EUS-FNA surpasses EUS-FNA with an EBUS bronchoscope in diagnostic ability. Although a few studies, ${ }^{13,26}$ ours among them, have included a few cases with successful EUS-
FNA for the station 5 lymph node, it cannot be assessed by EUS-FNA using an EBUS bronchoscope in most cases. The accessibility of conventional EUS-FNA for station 5 lymph nodes would be much better than that of EUS-FNA with an EBUS bronchoscope. In addition, the adrenal glands $^{32}$ or even station 6 lymph nodes ${ }^{33}$ can be potentially evaluated using conventional EUS-FNA. Although conventional EUS-FNA was not performed for any patients in our study, it might provide additional diagnostic information in certain cases. Nevertheless, the simplicity of EUS-FNA with an EBUS bronchoscope seems much more practical. One nonrandomized study that included 214 patients with lung cancer suggested that combined EBUS-TBNA and EUS-FNA with a single bronchoscope was equally effective and less time-consuming than combined EBUS-TBNA and conventional EUS-FNA. ${ }^{14}$ To resolve the issue regarding whether EUS-FNA with an EBUS bronchoscope can be substituted for that with an EUS endoscope, additional randomized studies are required.

The reason for adding EUS-FNA to EBUS-TBNA is to provide results for the lymph node stations that cannot be assessed using EBUS-TBNA. EUS-FNA can access station 8 or 9 or, occasionally, 5 lymph nodes, which are usually inaccessible using EBUS-TBNA. In addition, it could also

TABLE 8. Duration of procedures stratified by the number of lymph node stations sampled

\begin{tabular}{|c|c|c|c|c|c|c|c|c|c|c|}
\hline \multirow{3}{*}{$\begin{array}{c}\text { Lymph node stations } \\
\text { sampled (n) }\end{array}$} & \multicolumn{3}{|c|}{ EBUS } & \multicolumn{3}{|c|}{ EUS } & \multicolumn{3}{|c|}{ EBUS + EUS $*$} & \multirow[b]{3}{*}{$P$ value } \\
\hline & \multirow{2}{*}{ Patients (n) } & \multicolumn{2}{|c|}{ Procedure time (min) } & \multirow[b]{2}{*}{ Patients (n) } & \multicolumn{2}{|c|}{ Procedure time (min) } & \multirow[b]{2}{*}{ Patient (n) } & \multicolumn{2}{|c|}{ Procedure time (min) } & \\
\hline & & Median & Range & & Median & Range & & Median & Range & \\
\hline $0 \ddagger$ & $29(19)$ & 6.5 & $4.0-23.0$ & $41(28)$ & 3.5 & $1.3-14.0$ & $23(15)$ & 14.8 & $7.5-34.5$ & $<.01$ \\
\hline 1 & $40(27)$ & 13.9 & $9.0-36.5$ & $47(32)$ & 8.8 & $4.3-21.0$ & $37(25)$ & 22.5 & $12.8-39.5$ & $<.01$ \\
\hline 2 & $43(29)$ & 18.8 & $12.0-43.8$ & $52(35)$ & 12.5 & $8.3-28.0$ & $31(21)$ & 30.8 & $19.3-56.8$ & $<.01$ \\
\hline 3 & $26(17)$ & 20.8 & $14.8-41.0$ & $7(5)$ & 17 & $15.0-23.0$ & $35(23)$ & 34 & $21.8-63.8$ & .09 \\
\hline 4 & $9(6)$ & 32.3 & $23.0-42.0$ & $1(1)$ & 24 & 24.0 & $20(13)$ & 40.9 & $35.8-66.3$ & NA \\
\hline 5 & $1(1)$ & 36.8 & 36.8 & $0(0)$ & - & - & $1(1)$ & 44.3 & 44.3 & NA \\
\hline 6 & $2(1)$ & 32 & $29.5-34.5$ & $0(0)$ & - & - & $3(2)$ & 50.3 & $51-64.8$ & NA \\
\hline Total & $150(100)$ & 16.5 & $4.0-43.8$ & $148(100)$ & 10.1 & $1.3-28.0$ & $150(100)$ & 29 & $7.5-66.3$ & $<.01$ \\
\hline
\end{tabular}

$E B U S$, Endobronchial ultrasound; EUS, endoscopic ultrasound; $N A$, not available. *Duration from EBUS bronchoscope insertion into the trachea until removal from the esophagus. †EBUS versus EUS. †̦Examined by ultrasonography but not sampled because no target lesions were $>5 \mathrm{~mm}$. 
play an important role for some cases with other lymph nodes difficult to access using EBUS-TBNA, including station $2 \mathrm{~L}, 4 \mathrm{~L},{ }^{13,27}$ and 7 lymph nodes. ${ }^{6}$ The accessibility to station $4 \mathrm{~L}$ will be much better with EUS-FNA than with EBUS-TBNA in most cases. Even in station 7 lymph nodes, which have been reported as the most frequent location for EBUS-TBNA, the visibility of EUS has been superior to that of EBUS in some cases. Thus, additional EUS-FNA can be recommended, especially for patients with mediastinal lymph nodes that are inaccessible, difficult to access, or not clearly visualized from the airway.

To date, several investigators have suggested that EBUS-TBNA, which provides high sensitivity, can be an alternative to mediastinoscopy. ${ }^{3,34}$ Although our study was not designed to compare combined EBUS-TBNA and EUS-FNA with mediastinoscopy, the combined procedure seems to be reasonable as a first pathologic mediastinal staging test, because it can reduce the need for additional invasive surgical staging procedures. However, a substantial number of patients $(n=9)$ had false-negative EBUS-TBNA and EUS-FNA results in our study; thus, its role seems to be complementary, rather than an alternative, to surgical staging procedures.

A sequential EBUS and EUS examination in a single session seemed to be a well-tolerated and safe procedure. We could complete both procedures in all but 2 patients, who had developed a severe cough during EBUS-TBNA. No other complications were observed except for the minor usual ones associated with endoscopy, such as a minimal amount of blood from the puncture site, a mild cough, or pharyngeal discomfort at EBUS bronchoscope insertion. The present study was performed in the outpatient setting; thus, some minor self-healing complications that occurred after the procedures, including a low-grade fever, might have been underestimated. However, no patients required a specific treatment, including antibiotics for prophylaxis or treatment, in our study.

This was a single-center, nonrandomized study, which was a potential limitation. In a consecutive examination using a single bronchoscope, the order of the transbronchial approach followed by the transesophageal approach seemed reasonable to minimize the risk of infection; however, it could have affected the accuracy and safety of each procedure. Furthermore, it is well-known that the yield of endoscopic procedures largely depends on the examiner's experience and skill. Our results might not be readily duplicated by less experienced examiners, and better results might be achieved by more skilled examiners. Another limitation was the reliability of the final diagnosis. In the present study, 34 patients had no surgical confirmation of $\mathrm{N} 2$ and N3 disease. Thus, the risk exists of inaccuracy if the reference standard is used for referent values. In addition, not all hilar or mediastinal lymph nodes were explored during surgery, which could have led to an overestimation of the endoscopic diagnostic value. However, it would have affected the diagnostic value of each procedure equally; thus, our conclusions regarding the significant superiority of the combined method are well founded.

$\mathrm{We}$, therefore, consider that the combined endoscopic approach with EBUS-TBNA and EUS-FNA with a single bronchoscope is an accurate and safe method for preoperative hilar and mediastinal staging of NSCLC and better than each technique alone.

\section{References}

1. Silvestri GA, Gonzalez AV, Jantz MA, Margolis ML, Gould MK, Tanoue LT, et al. Methods for staging non-small cell lung cancer: diagnosis and management of lung cancer, 3rd ed: American College of Chest Physicians evidence-based clinical practice guidelines. Chest. 2013;143(5 Suppl):e211S-50S.

2. Ernst A, Anantham D, Eberhardt R, Krasnik M, Herth FJ. Diagnosis of mediastinal adenopathy-real-time endobronchial ultrasound guided needle aspiration versus mediastinoscopy. J Thorac Oncol. 2008;3:577-82.

3. Yasufuku K, Pierre A, Darling G, de Perrot M, Waddell T, Johnston M, et al. A prospective controlled trial of endobronchial ultrasound-guided transbronchial needle aspiration compared with mediastinoscopy for mediastinal lymph node staging of lung cancer. J Thorac Cardiovasc Surg. 2011;142:1393-400.

4. Annema JT, Versteegh MI, Veseliç M, Welker L, Mauad T, Sont JK, et al. Endoscopic ultrasound added to mediastinoscopy for preoperative staging of patients with lung cancer. JAMA. 2005;294:931-6.

5. Tournoy KG, De Ryck F, Vanwalleghem LR, Vermassen F, Praet M, Aerts JG, et al. Endoscopic ultrasound reduces surgical mediastinal staging in lung cancer: a randomized trial. Am J Respir Crit Care Med. 2008;177:531-5.

6. Rintoul RC, Skwarski KM, Murchison JT, Wallace WA, Walker WS, Penman ID. Endobronchial and endoscopic ultrasound-guided real-time fine-needle aspiration for mediastinal staging. Eur Respir J. 2005;25:416-21.

7. Vilmann P, Krasnik M, Larsen SS, Jacobsen GK, Clementsen P. Transesophageal endoscopic ultrasound-guided fine-needle aspiration (EUS-FNA) and endobronchial ultrasound-guided transbronchial needle aspiration (EBUSTBNA) biopsy: a combined approach in the evaluation of mediastinal lesions. Endoscopy. 2005;37:833-9.

8. Wallace MB, Pascual JM, Raimondo M, Woodward TA, McComb BL, Crook JE, et al. Minimally invasive endoscopic staging of suspected lung cancer. JAMA. 2008;299:540-6.

9. Szlubowski A, Zieliński M, Soja J, Annema JT, Sośnicki W, Jakubiak M, et al. A combined approach of endobronchial and endoscopic ultrasound-guided needle aspiration in the radiologically normal mediastinum in non-small-cell lung cancer staging - a prospective trial. Eur J Cardiothorac Surg. 2010;37:1175-9.

10. Ohnishi R, Yasuda I, Kato T, Tanaka T, Kaneko Y, Suzuki T, et al. Combined endobronchial and endoscopic ultrasound-guided fine needle aspiration for mediastinal nodal staging of lung cancer. Endoscopy. 2011;43:1082-9.

11. Tournoy KG, Annema JT, Krasnik M, Herth FJ, van Meerbeeck JP. Endoscopic and endobronchial ultrasonography according to the proposed lymph node map definition in the seventh edition of the tumor, node, metastasis classification for lung cancer. J Thorac Oncol. 2009;4:1576-84.

12. Herth FJ, Krasnik M, Kahn N, Eberhardt R, Ernst A. Combined endoscopicendobronchial ultrasound-guided fine-needle aspiration of mediastinal lymph nodes through a single bronchoscope in 150 patients with suspected lung cancer. Chest. 2010;138:790-4.

13. Hwangbo B, Lee GK, Lee HS, Lim KY, Lee SH, Kim HY, et al. Transbronchial and transesophageal fine-needle aspiration using an ultrasound bronchoscope in mediastinal staging of potentially operable lung cancer. Chest. 2010;138: 795-802.

14. Szlubowski A, Soja J, Kocon P, Talar P, Czajkowski W, Rudnicka-Sosin L, et al. A comparison of the combined ultrasound of the mediastinum by use of a single ultrasound bronchoscope versus ultrasound bronchoscope plus ultrasound gastroscope in lung cancer staging: a prospective trial. Interact Cardiovasc Thorac Surg. 2012;15:442-6.

15. Goldstraw P, Crowley J, Chansky K, Giroux DJ, Groome PA, Rami-Porta R, et al; International Association for the Study of Lung Cancer International Staging Committee; Participating Institutions. The IASLC Lung Cancer Staging Project: proposals for the revision of the TNM stage groupings in the forthcoming 
(seventh) edition of the TNM classification of malignant tumours. $J$ Thorac Oncol. 2007;2:706-14.

16. Rusch VW, Asamura H, Watanabe H, Giroux DJ, Rami-Porta R, Goldstraw P. Members of IASLC Staging Committee. The IASLC lung cancer staging project: a proposal for a new international lymph node map in the forthcoming seventh edition of the TNM classification for lung cancer. J Thorac Oncol. 2009;4: 568-77.

17. Oki M, Saka H, Kitagawa C, Kogure Y, Murata N, Ichihara S, et al. Prospective study of endobronchial ultrasound-guided transbronchial needle aspiration of lymph nodes versus transbronchial lung biopsy of lung tissue for diagnosis of sarcoidosis. J Thorac Cardiovasc Surg. 2012;143:1324-9.

18. Herth FJ, Eberhardt R, Vilmann P, Krasnik M, Ernst A. Real-time endobronchial ultrasound guided transbronchial needle aspiration for sampling mediastinal lymph nodes. Thorax. 2006;61:795-8.

19. Oki M, Saka H, Kitagawa C, Kogure Y, Murata N, Adachi T, et al. Transesophageal bronchoscopic ultrasound-guided fine needle aspiration for diagnosis of sarcoidosis. Respiration. 2013;85:137-43.

20. Nakajima T, Yasufuku K, Iyoda A, Yoshida S, Suzuki M, Sekine Y, et al. The evaluation of lymph node metastasis by endobronchial ultrasound-guided transbronchial needle aspiration: crucial for selection of surgical candidates with metastatic lung tumors. J Thorac Cardiovasc Surg. 2007;134:1485-90.

21. Zielinski M, Szlubowski A, Kołodziej M, Orzechowski S, Laczynska E, Pankowski J, et al. Comparison of endobronchial ultrasound and/or endoesophageal ultrasound with transcervical extended mediastinal lymphadenectomy for staging and restaging of non-small-cell lung cancer. J Thorac Oncol. 2013;8:630-6.

22. Tournoy KG, Keller SM, Annema JT. Mediastinal staging of lung cancer: novel concepts. Lancet Oncol. 2012;13:e221-9.

23. Annema JT, van Meerbeeck JP, Rintoul RC, Dooms C, Deschepper E, Dekkers OM, et al. Mediastinoscopy vs endosonography for mediastinal nodal staging of lung cancer: a randomized trial. JAMA. 2010;304:2245-52.

24. Zhang R, Ying K, Shi L, Zhang L, Zhou L. Combined endobronchial and endoscopic ultrasound-guided fine needle aspiration for mediastinal lymph node staging of lung cancer: a meta-analysis. Eur J Cancer. 2013;49: 1860-7.
25. Medford AR, Agrawal S. Single bronchoscope combined endoscopicendobronchial ultrasound-guided fine-needle aspiration for tuberculous mediastinal nodes. Chest. 2010;138:1274.

26. Hwangbo B, Lee HS, Lee GK, Lim KY, Lee SH, Kim HY, et al Transoesophageal needle aspiration using a convex probe ultrasonic bronchoscope. Respirology. 2009;14:843-9.

27. Oki M, Saka H, Kitagawa C, Sato S. Bronchoscopic transesophageal ultrasound-guided needle aspiration: an alternative to the conventional transesophageal ultrasound-guided needle aspiration technique. J Thorac Cardiovasc Surg. 2010;139:1659-61.

28. Oki M, Saka H, Kitagawa C. Transesophageal bronchoscopic ultrasound-guided fine-needle aspiration for diagnosis of peripheral lung cancer. Ann Thorac Surg. 2011;91:1613-6.

29. Sharples LD, Jackson C, Wheaton E, Griffith G, Annema JT, Dooms C, et al. Clinical effectiveness and cost-effectiveness of endobronchial and endoscopic ultrasound relative to surgical staging in potentially resectable lung cancer: results from the ASTER randomised controlled trial. Health Technol Assess. 2012;16:1-75, iii-iv.

30. Lee HS, Lee GK, Lee HS, Kim MS, Lee JM, Kim HY, et al. Real-time endobronchial ultrasound-guided transbronchial needle aspiration in mediastina staging of non-small cell lung cancer: how many aspirations per target lymph node station? Chest. 2008;134:368-74.

31. Block MI. Endobronchial ultrasound for lung cancer staging: how many stations should be sampled? Ann Thorac Surg. 2010;89:1582-7.

32. Uemura S, Yasuda I, Kato T, Doi S, Kawaguchi J, Yamauchi T, et al Preoperative routine evaluation of bilateral adrenal glands by endoscopic ultrasound and fine-needle aspiration in patients with potentially resectable lung cancer. Endoscopy. 2013;45:195-201.

33. Liberman M, Duranceau A, Grunenwald E, Thiffault V, Khereba M, Ferraro P. Initial experience with a new technique of endoscopic and ultrasonographic access for biopsy of para-aortic (station 6) mediastinal lymph nodes without traversing the aorta. J Thorac Cardiovasc Surg. 2012;144:787-92.

34. Lee BE, Kletsman E, Rutledge JR, Korst RJ. Utility of endobronchial ultrasoundguided mediastinal lymph node biopsy in patients with non-small cell lung cancer. J Thorac Cardiovasc Surg. 2012;143:585-90.

\title{
EDITORIAL COMMENTARY
}

\section{Pathologic staging of the mediastinum: When and how?}

\author{
Jacob A. Klapper, MD, and Chadrick E. Denlinger, MD
}

Pathologic staging of mediastinal lymph nodes before surgical resection is the accepted standard for patients with non-small cell lung cancer (NSCLC). ${ }^{1}$ Mediastinoscopy, endobronchial ultrasound (EBUS), and endoscopic ultrasound are all acceptable means of obtaining tissue. Two prospective studies compared the sensitivity of EBUS with mediastinoscopy among patients with NSCLC

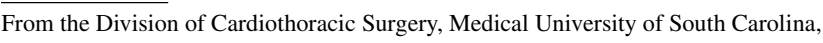
Charleston, SC.

Disclosures: Authors have nothing to disclose with regard to commercial support.

Address for reprints: Chadrick E. Denlinger, MD, 25 Courtenay Dr, ART Ste 7018,

Charleston, SC 29425. (E-mail: denlinge@musc.edu).

J Thorac Cardiovasc Surg 2014;148:1177-8

0022-5223/\$36.00

Copyright (c) 2014 by The American Association for Thoracic Surgery

http://dx.doi.org/10.1016/j.jtcvs.2014.09.001
}

and concluded that the 2 modalities are equivalent. ${ }^{2,3}$ In the first study patients were randomized to either mediastinoscopy or EBUS followed by mediastinoscopy if EBUS was negative. The sensitivity of mediastinoscopy alone was $79 \%$ compared with $85 \%$ for EBUS. The addition of mediastinoscopy in patients previously evaluated by EBUS increased the sensitivity to $94 \%{ }^{2}$ In the second study, by Yasufuku and colleagues, ${ }^{3}$ each patient was evaluated by both EBUS and mediastinoscopy and the sensitivities of the 2 were $81 \%$ and $79 \%$, respectively.

The sensitivity of $73 \%$ for combined EBUS and endoscopic ultrasound presented by Oki and colleagues ${ }^{4}$ in this issue is congruent with prior surgical series where patients went on to resection if the mediastinum was 


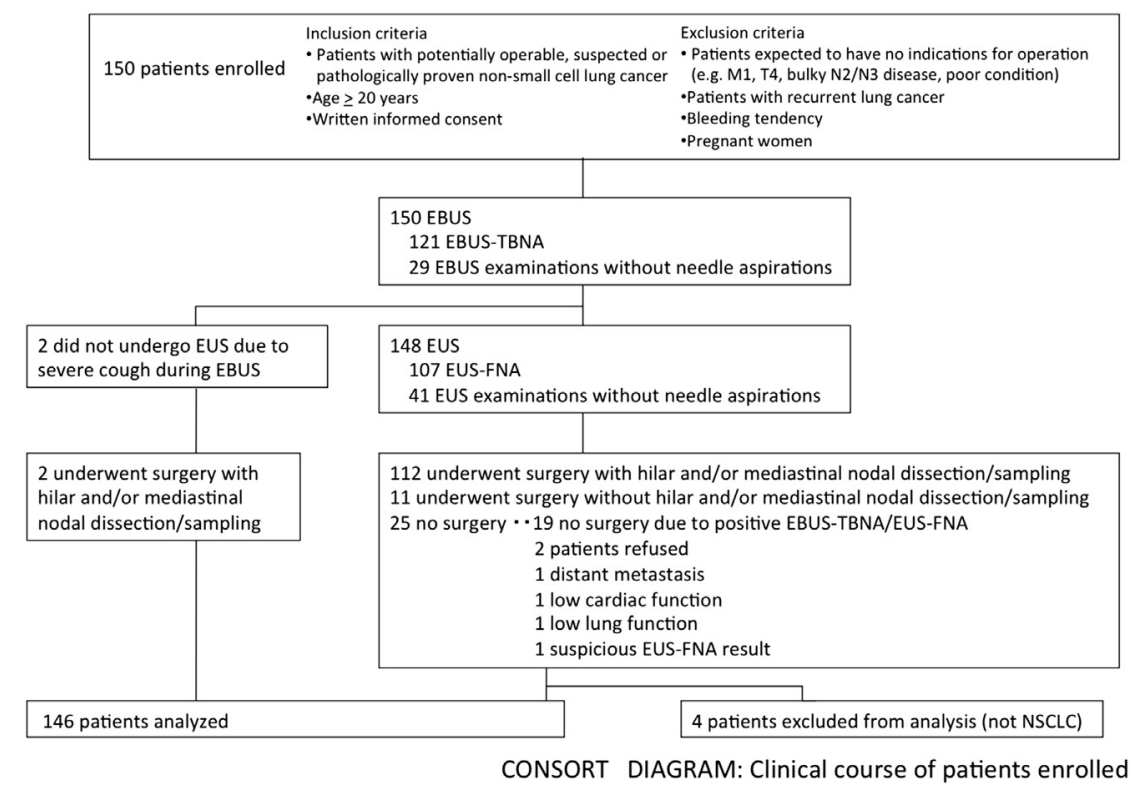

FIGURE E1. CONSORT diagram showing the clinical course of patients enrolled in the study. 